\title{
Altered sensory feedbacks in pianist's dystonia: the altered auditory feedback paradigm and the glove effect
}

\author{
Felicia P.-H. Cheng, Michael Großbach and Eckart O. Altenmüller* \\ Institute of Music Physiology and Musicians' Medicine, Hanover University of Music, Drama, and Media, Hannover, Germany
}

\section{Edited by:}

Robert J. Zatorre, McGill University,

Canada

Reviewed by:

Shanqing Cai, Boston University, USA

Peter Pfordresher, University at Buffalo State University of New York, USA

\section{*Correspondence:}

Eckart O. Altenmüller, Institute of Music Physiology and Musicians Medicine, Hanover University of Music, Drama, and Media,

Emmichplatz 1, 30175 Hannover, Germany

e-mail: eckart.altenmueller@ hmtm-hannover.de
Background: This study investigates the effect of altered auditory feedback (AAF) in musician's dystonia (MD) and discusses whether AAF can be considered as a sensory trick in MD. Furthermore, the effect of AAF is compared with altered tactile feedback, which can serve as a sensory trick in several other forms of focal dystonia.

Methods: The method is based on scale analysis (Jabusch et al., 2004). Experiment 1 employs synchronization paradigm: $12 \mathrm{MD}$ patients and 25 healthy pianists had to repeatedly play $\mathrm{C}$-major scales in synchrony with a metronome on a MIDI-piano with three auditory feedback conditions: (1) normal feedback; (2) no feedback; (3) constant delayed feedback. Experiment 2 employs synchronization-continuation paradigm: $12 \mathrm{MD}$ patients and 12 healthy pianists had to repeatedly play C-major scales in two phases: first in synchrony with a metronome, secondly continue the established tempo without the metronome. There are four experimental conditions, among them three are the same AAF as in Experiment 1 and 1 is related to altered tactile sensory input. The coefficient of variation of inter-onset intervals of the key depressions was calculated to evaluate fine motor control.

Results: In both experiments, the healthy controls and the patients behaved very similarly. There is no difference in the regularity of playing between the two groups under any condition, and neither did AAF nor did altered tactile feedback have a beneficial effect on patients' fine motor control.

Conclusions: The results of the two experiments suggest that in the context of our experimental designs, AAF and altered tactile feedback play a minor role in motor coordination in patients with musicians' dystonia. We propose that altered auditory and tactile feedback do not serve as effective sensory tricks and may not temporarily reduce the symptoms of patients suffering from MD in this experimental context.

Keywords: musician's dystonia, altered auditory feedback, glove effect, sensory trick, scale paradigm, sensorimotor integration

\section{INTRODUCTION}

Dystonia in pianists belongs to a group of dystonic movement disorders termed focal hand dystonias (Chen and Hallett, 1998). It is characterized by the degradation of voluntary control of highly skilled movement patterns involved in piano playing. The condition frequently results in co-contraction of wrist flexors and extensors and in involuntary curling, or extending of digits, thus rendering fast movements involved for example in scale playing irregular (Jabusch et al., 2004). It is known that focal hand dystonia involves several sensory abnormalities, such as reduced two-point discrimination thresholds, reduced graphaesthesia (Byl et al., 1996), and an impairment of the thermal detection thresholds (Suttrup et al., 2011). Furthermore, temporal judgment of somatosensory and auditory stimuli has been shown to be altered in musicians suffering from dystonia (Lim et al., 2003). On the other hand, cutaneous stimuli may reduce the severity of motor symptoms in some forms of focal dystonia. This phenomenon is termed sensory trick. Studies into the "geste antagoniste," a sensory trick reducing severity of Torticollis, (a focal dystonia causing involuntary movements of the muscles of neck and shoulder) suggested that successful sensory tricks can be regarded as perceptual dysbalance, induce increased activation of the parietal cortex and together with the frontal cortex, mediates distinct sensorimotor transformations that can help with correcting the long-term dystonic posture. Accordingly, successful sensory tricks should be regarded as a complex dynamic mechanism that corrects the perceptual dysbalance of the abnormally defined posture (Naumann et al., 2000; Schramm et al., 2004). In task-specific focal dystonia, sensory stimuli like wearing a latex glove may also reduce the severity of motor symptoms in some forms of focal dystonia (Jabusch et al., 2011).

Based on the models proposed by the previous sensory trick studies, the present study aimed at investigating the effect of altered auditory feedback (AAF) on musician's dystonia (MD) under two widely used paradigms for studying motor behavior: (1) synchronization paradigm; (2) synchronization-continuation paradigm. The motivation for this study came from anecdotal reports of organ players suffering from hand dystonia who 
reported a marked improvement of the motor symptoms when playing on a pipe organ with delayed sound production after the key stroke due to mechanical coupling of the keyboard and the organ pipes. Such observation was valuable for the movement disorders research, yet the possible influence of AAF and altered somatosensory feedback on the fine motor control in MD had never been investigated. Considering the delayed auditory feedback in these reports, it is well known that the extensive training of professional musicians leads to plastic adaptations of neural networks involved in the demanding temporal-spatial control of overtrained movements (Bangert et al., 2006.; Zatorre et al., 2007), and it has been shown that AAF has a great influence on the motor control in music performance of healthy musicians (Pfordresher, 2006). Furthermore, it has been shown that AAF can induce improvement in speech in some movement disorders and motor speech disorders such as stuttering, dysarthria and Parkinson's disease (Downie et al., 1981; Gentil, 1993; Alm, 2004). Based on the above reasons, we therefore hypothesized that AAF could account for the anecdotal observations of the organ players and have an influence on the dystonic symptoms, similar to the cutaneous sensory trick in torticollis patients.

\section{MATERIALS AND METHODS}

All procedures were approved by the ethics committee of Hannover Medical School and participants gave written informed consent before data collection. All patients suffering from MD were recruited from the outpatient clinic of the Institute of Music Physiology and Musicians' Medicine of the Hannover University of Music, Drama and Media. They underwent complete neurological examination and were diagnosed by one of the authors, who is a neurologist and movement disorders specialist (EA). All other neurological and health issues were excluded.

\section{EXPERIMENT 1: SYNCHRONIZATION STUDY Participants}

Twelve professional pianists ( 8 males, 4 females, mean age $=$ $44.5, S D=9.6$, mean accumulated practice time $=42,086 \mathrm{~h}$, $S D=25,040)$ suffering from right hand MD and 25 healthy professional pianists ( 13 males, 12 females, mean age $=25.8$ years old, $S D=3.93$, mean accumulated practice time $=25,075 \mathrm{~h}$, $S D=10,754)$ participated in this experiment. Combined two one-sided $t$-tests (TOST, Robinson and Froese, 2004) shows that the two groups are not equivalent with regard of their accumulated practice hours $(p=0.92)$. According to the Edinburgh inventory (Oldfield, 1971), all the patients were right handed. Twenty four of the healthy professional pianists were right handed and one was left handed.

\section{Procedure}

The method is based on Scale Analysis (Jabusch et al., 2004). Subjects were instructed to repeatedly play 2 octaves of $\mathrm{C}$ major scales (from C4 to C6) in legato-style at a tempo of 80 beats per minute with four notes per beat (inter-onset intervals $=187.5 \mathrm{~ms}$ ) in both upward and downward directions as accurately as possible on a MIDI digital piano (Wersi Digital Piano CT2) with their right hand only. There were three conditions for different types of auditory feedback: (1) normal feedback (NORMAL), in which the auditory feedback occurred simultaneously with the key depression; (2) no feedback (MUTE), in which no auditory feedback is produced with the key depression; (3) fixed delayed feedback of $200 \mathrm{~ms}$ (DELAY200), in which the auditory feedback occurred $200 \mathrm{~ms}$ after the key depression. The selection of delay duration was meant to mimick the delayed auditory feedback of a pipe organ in a resonant space. For each condition, the participant had to play at least 25 times of complete upward and downward scales. The order of the conditions was randomized, and all playing was synchronized to a real metronome placed on the digital piano (Wittner metronome QM2 taktell). In DELAY200, subjects were explicitly instructed to synchronize the piano sound to the metronome, not the movement. The onset and the offset time of each key depression was recorded (measured in milliseconds, start of the program is defined as $0 \mathrm{~ms}$ ). MIDI recording, as well as manipulation for the conditions MUTE and DELAY, were done using a custom-made $\mathrm{C}$ program which acquired and (where applicable) manipulated the MIDI events coming from the MIDI piano. Sound was played back to subjects via the computer sound chip and two studio monitor speakers (Yamaha MSP 5) placed approximately $1 \mathrm{~m}$ in front of the subjects, $150 \mathrm{~cm}$ apart.

\section{Data analysis}

For each condition, as a measure of evenness of piano playing (which is required in professional pianists), the regularity of timing of successive keystrokes (termed inter-onset intervals, abbreviated as IOIs) was calculated from at least 20 sets of complete scales, except for the last IOI of every scale because it was frequently elongated according to the pianist's expressive playing. The coefficients of variation (CVs) were calculated to indicate the irregularity of timing of the scale playing. The CV is defined as: $\hat{c}_{v}^{*}=\left(1+\frac{1}{4 n}\right) \frac{s}{x}$, where $s$ is the standard deviation of the measured IOIs, $x$ is the mean of the measured IOIs and $n$ is the number of scales a participant played in a given condition (Sokal and Rohlf, 1995) and with this definition the term $(1+1 / 4 n)$ approaches 1 asymptotically with increasing $n$. The IOI CVs were computed for upward and downward scales individually since these are two different motor patterns. Finally the IOI CVs for all the patients and participants under all types of auditory feedback and for both upward and downward directions of scale playing were analyzed with ANOVA to detect the main effect of group and condition. The within factors are playing direction (with levels "up" and "down") and types of auditory feedback (with levels "NORMAL," "MUTE," and "DELAY200"); the between factor is group (with levels "patient" and "control"). The means of IOIs for both playing directions under different conditions have been reported as well. All analyses were done using R (version 2.15.2; R Core Team, 2005) scripts in RStudio (version 0.97.551; RStudio, 2013). Multiple comparisons were corrected using Holm's (1979) method.

\section{RESULTS OF EXPERIMENT 1}

The target dependent variable in this study was the coefficient of variation of inter-onset intervals, being an objective measure of playing regularity in pianists with MD. To determine differences in playing speed, the inter-onset intervals were analyzed as well: regarding to the mean IOIs, a main effect of auditory feedback 
condition $\left[F_{(2,70)}=8.832\right.$, Greenhouse-Geisser epsilon $=0.740$, $p<0.001]$ was found but no effect of group $\left[F_{(1,35)}=0.350\right.$, $p>0.05]$ or scale playing direction $\left[F_{(1,35)}=2.445, p=0.127\right]$ and no significant interactions were found $\left[F_{(2,70)}=2.498\right.$, $p>0.05$ ] (see Figure 1). The results of the healthy group during DELAY200 condition were untypical comparing to most reports of delayed auditory feedback. In our results of Experiment 1, the healthy group showed a decrease in mean IOIs (movement speeding up) while in most reports the participants show increased IOIs (movement slowing down). Possible explanations for this result are (1) as a strategy to cope with the delayed auditory feedback, the participants played faster in order to "get ahead" of the delay (Gates et al., 1974); (2) the effect of delayed auditory feedback on the timing of movement production is related to both the phase of actual movement execution and cognitive planning. It has been suggested that when delayed auditory feedback co-occurs with the downswing phase of movement execution, on a cognitive planning level, auditory information compliments the regulation of movement, and thus facilitates the approach to the goal, which may shorten the IOIs (Pfordresher and Dalla Bella, 2011). This effect might had played a role in our Experiment 1.

Regarding to the IOI CVs, there was a main effect of group $\left[F_{(1,35)}=16.427, p<0.001\right]$ and the patient group generally showed higher IOI CVs than the healthy controls. A main effect of feedback condition was also found $\left[F_{(2,70)}=34.178\right.$, Greenhouse-Geisser epsilon $=0.676, p<0.001]$, but no significant interactions were found (Figure 2). Both groups had increased IOI CVs for the DELAY200 condition, showing that DELAY200 induced unevenness in scale playing (pairwise $t$-tests:

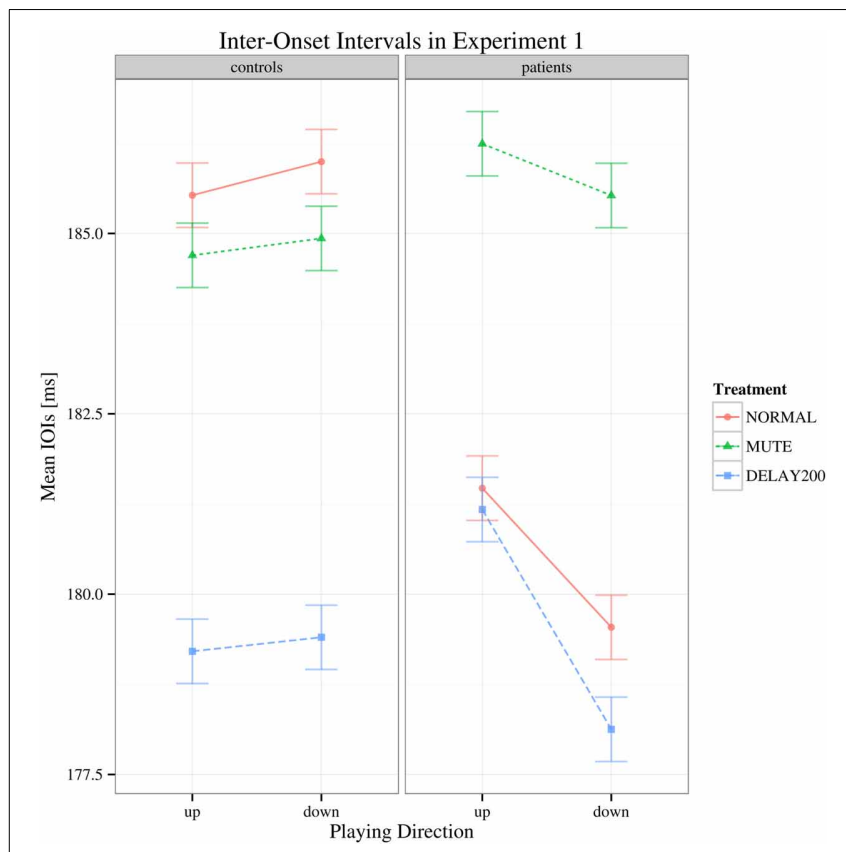

FIGURE 1 | Interaction plot of mean IOI's for Experiment 1. Shown are the estimated mean IOls (ms) conditional on group membership and auditory manipulation, collapsed over playing direction. Error bars denote 95\% confidence intervals.
NORMAL-MUTE: $p>0.05$; NORMAL-DELAY200: $p<0.001$; DELAY200-MUTE: $p<0.001$ ), meaning that they did not benefit from these two auditory manipulations in this synchronization paradigm. The patient group showed a very weak tendency toward higher IOI CVs in upward scale playing [Figure 2; $\left.F_{(1,35)}=3.497, p=0.07\right]$. This is possibly related to the taskspecificity of focal hand dystonia revealed under different motor patterns involved in playing directions. It should be mentioned, that the upwards scales are more complex due to the thumbunder passage, requiring a complex anticipatory coordination maneuver of thumb, remaining four fingers and the wrist.

\section{DISCUSSION OF EXPERIMENT 1}

The results of Experiment 1 showed that neither did the complete deprivation of auditory feedback nor did a constantly delayed auditory feedback of $200 \mathrm{~ms}$ serve as a successful sensory trick, which is against our hypothesis. Experiment 1 also showed an effect of auditory feedback condition on mean IOIs in both groups. However, the two groups of participants are very different in terms of their age and their years of accumulated hours of practice. Pianists suffering from MD are generally older than the healthy controls. In Experiment 2, we had tried our best to reduce this discrepancy.

\section{EXPERIMENT 2: SYNCHRONIZATION-CONTINUATION STUDY}

Following Experiment 1, in which deprivation of auditory feedback and delayed auditory feedback did not serve as a sensory trick that could improve the fine motor control in MD, Experiment 2 was carried out. One problem inherent to playing scales with delayed feedback to a metronome is probably the higher task difficulty in this condition. Anticipating the auditory

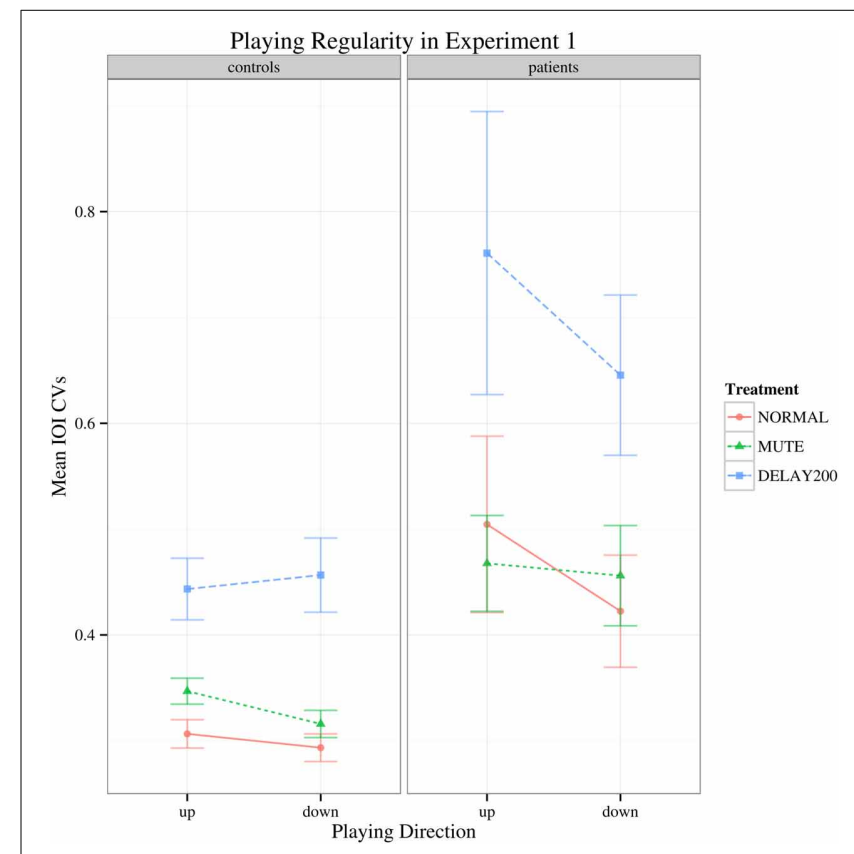

FIGURE 2 | Interaction plot of IOI CVs for Experiment 1. Error bars denote $95 \%$ confidence intervals. 
feedback and executing the key presses $200 \mathrm{~ms}$ in advance during scale playing in order to exactly synchronize with the metronome proved to be difficult for both healthy pianists and pianists suffering from $\mathrm{MD}$, and this might have shifted the pianist's attention away from the scale playing toward the out-of-phase synchronization of movements to the metronome, leading to a different neural process that is required to pace the movement according to an external stimulus (Serrien, 2008). To circumvent this problem, in Experiment 2 we applied the synchronization-continuation paradigm, and reduced the delay to $90 \mathrm{~ms}$ since this would reduce the degree of asynchrony involved in Experiment 1.

\section{Participants}

Twelve professional pianists suffering from right hand MD (9 males, 3 females, mean age $=41, S D=10.4$, mean accumulated practice time $=37,593 \mathrm{~h}, S D=11199)$ and 12 healthy professional pianists ( 5 males, 7 females, mean age $=33.3$ years old, $S D=11.6$, mean accumulated practice time $=35,495 \mathrm{~h}, S D=$ $24,317)$ participated in this experiment. TOST suggested that the two groups were not equivalent with regard of their accumulated practice hours $(p=0.356)$. All the pianists suffering from $\mathrm{MD}$ were right handed. Eleven of the healthy controls were right handed and 1 was left handed, according to the Edinburgh inventory (Oldfield, 1971). Two of the pianists suffering from right hand MD had participated in Experiment 1. None of the healthy pianists had participated in Experiment 1.

\section{Procedure}

Similar to Experiment 1, the method of Experiment 2 is also based on Scale Analysis, but the conditions are designed according to the synchronization-continuation paradigm (Stevens, 1886; Finney and Warren, 2002; Pfordresher and Palmer, 2002), and one more condition, which is the altered tactile feedback condition, was included. The synchronization-continuation paradigm is widely used in studies of temporal coordination between actions and sound. During the experiment, the participants were instructed to repeatedly play 2 octaves of $\mathrm{C}$ major scales (from $\mathrm{C} 4$ to $\mathrm{C} 6$ ) with right hand in legato-style at a tempo of 80 beats per minute with 16 th notes (four notes per beat, inter-onset interval $=187.5 \mathrm{~ms}$ ) in either upward or downward directions as accurate as possible on the same digital piano used in Experiment 1. The first phase of each condition was the synchronization part, the participant had to play 10 sets of scales according to a metronome click sound generated by the computer and played through two speakers (Yamaha MSP 5). The second phase of each condition was the continuation part and the participant had to maintain the same way and same tempo of playing for at least 20 sets of complete and error-free scales in the absence of metronome sound. It was in the continuation part that the AAF (if any) was employed. Similar to Experiment 1, there were three different types of auditory feedback used in the continuation part: (1) normal auditory feedback (NORMAL); (2) muted feedback (MUTE); (3) with fixed delayed feedback of $90 \mathrm{~ms}$ (DELAY90). In the (4) tactile condition, auditory feedback was as in NORMAL but subjects wore a latex glove (GLOVE) on their playing hand throughout both the synchronization and continuation phases. The order of the conditions was randomized in two blocks. In one block the participants played upward scales only, and in the other block they played downward scales only. The order of the two blocks was also randomized. Between the blocks the participants were instructed to take a 2 min rest. The metronome sound onset time in the synchronization phase and the onset and the offset time of each key depression were recorded (measured in milliseconds, start of the program is defined as $0 \mathrm{~ms}$ ). Other than that, apparatus was the same as in Experiment 1.

\section{Data analysis}

Both the synchronization and the continuation parts of each condition were analyzed. The first scale of the continuation phase was discarded from the analysis because of the common increase in unevenness among the participants. Similar to Experiment 1, the IOI CVs from at least 20 sets of complete, error-free scales were calculated to indicate the irregularity of timing of the participants in each condition. To determine how well subjects synchronized with the metronome, we ran a within-between ANOVA on the mean temporal deviation of key presses from the metronome tick [between factor: group (levels "patient" and "control"); withinfactors: treatment (levels "NORMAL," "MUTE," "DELAY90," "GLOVE") and playing direction (levels "up," "down")]. On the IOI CVs, again a mixed effects ANOVA was calculated (factors and levels same as just mentioned). After the results suggested that there was no effect of playing direction, a between-within Ss ANOVA was run on the mean IOI CVs collapsed across playing directions to increase possible group effects [between effect: group (levels "control," "patient"), within effect: treatment (levels "NORMAL," "MUTE," "DELAY90," “GLOVE”)]. All analyses were done using R (version 2.15.2; R Core Team, 2005) scripts in RStudio (version 0.97.551; RStudio, 2013).

\section{RESULTS OF EXPERIMENT 2}

For the synchronization phase, both groups display negative asynchrony (keypress was in advance of the metronome; Repp, 1999). No significant main effect of group was found in this phase, suggesting that there is no difference between the patient and control group in terms of synchronization $\left[F_{(1,22)}=2.679, p>\right.$ 0.05; Figure 3], showing that both groups had similar degree of synchronization to the metronome. There were also no statistically significant effects of sensory feedback $\left[F_{(3,66)}=0.877\right.$, Greenhouse-Geisser epsilon $=0.513, p>0.05]$ and playing direction $\left[F_{(1,22)}=2.807, p>0.05\right]$.

For the continuation phase, regarding to the mean IOI's, no main effects and no significant interactions were found [group: $F_{(1,22)}=1.726, p>0.05$; sensory feedback: $F_{(3,66)}=$ 3.069, Greenhouse-Geisser epsilon $=0.529, p>0.05$; playing direction: $F_{(1,22)}=0.001, p>0.05$; group $\times$ sensory feedback: $\left.F_{(3,66)}=0.772, p>0.05\right)$ (Figure 4).

Similar to Experiment 1, with regard to the IOI CVs, a significant main effect was found for sensory feedback $\left[F_{(3,66)}=\right.$ 93.026, Greenhouse-Geisser epsilon $=0.385, p<0.001]$, possibly attributable to a significantly deteriorated motor performance for DELAY condition during the continuation phase (pairwise $t$-tests: NORMAL-MUTE: $p>0.05$; NORMAL-DELAY90: $p \ll 0.05$; NORMAL-GLOVE: $p>0.05$; Figure 5). No significant interactions were found $\left[F_{(3,66)}=1.65, p>0.05\right]$. Individual 


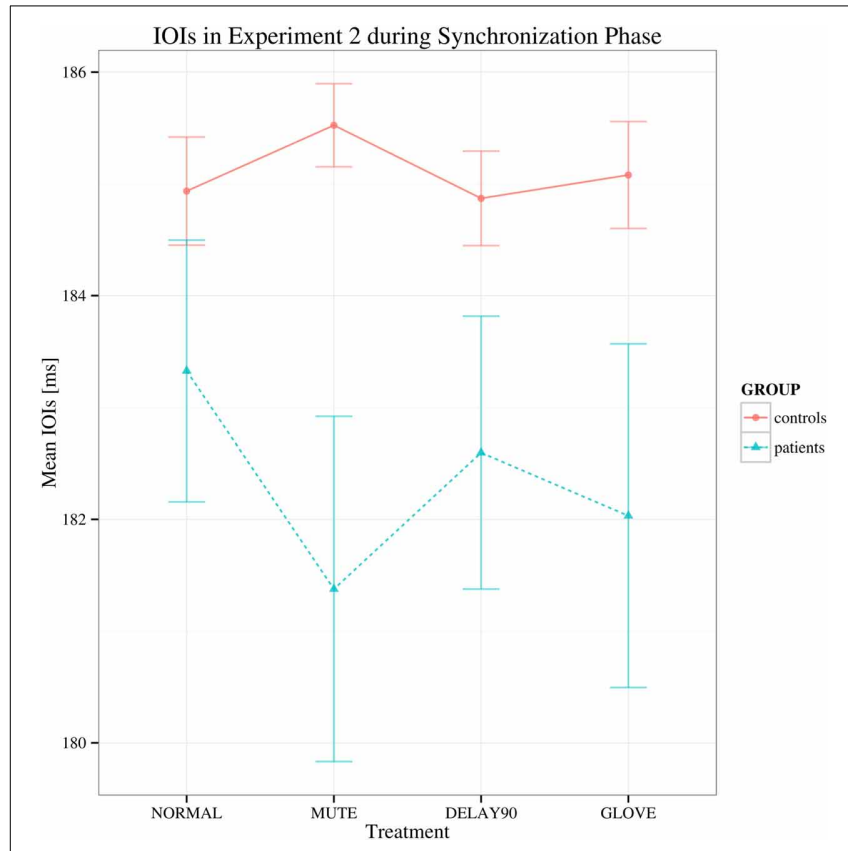

FIGURE 3 | Mean of the mean deviations from the metronome in the synchronization phase in Experiment 2. Shown are the estimated mean IOls (ms) conditional on group membership and auditory manipulation, collapsed over playing directions. Error bars denote $95 \%$ confidence intervals.

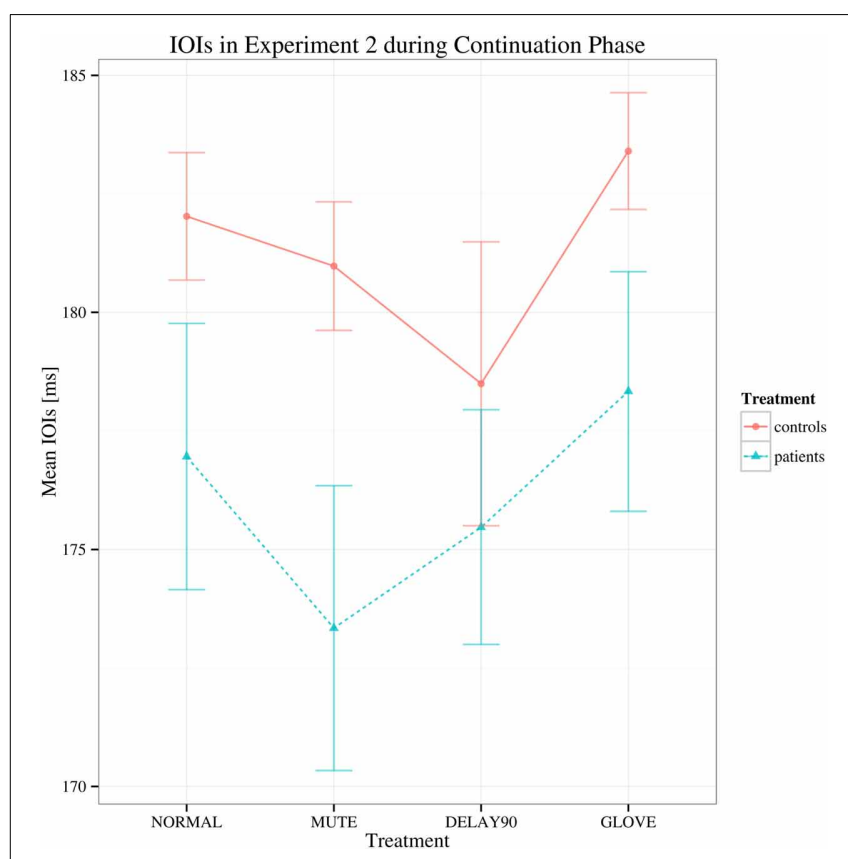

FIGURE 4 | Interaction plot of mean IOls for Experiment 2 during continuation phase. Details as in Figure 1. Error bars denote $95 \%$ confidence intervals.

mean IOI CVs of all the patients during the continuation phase under different conditions are detailed in Table 1, with the IOI CVs of upward and downward directions averaged. From the table, one can see that only four patients who have less severe

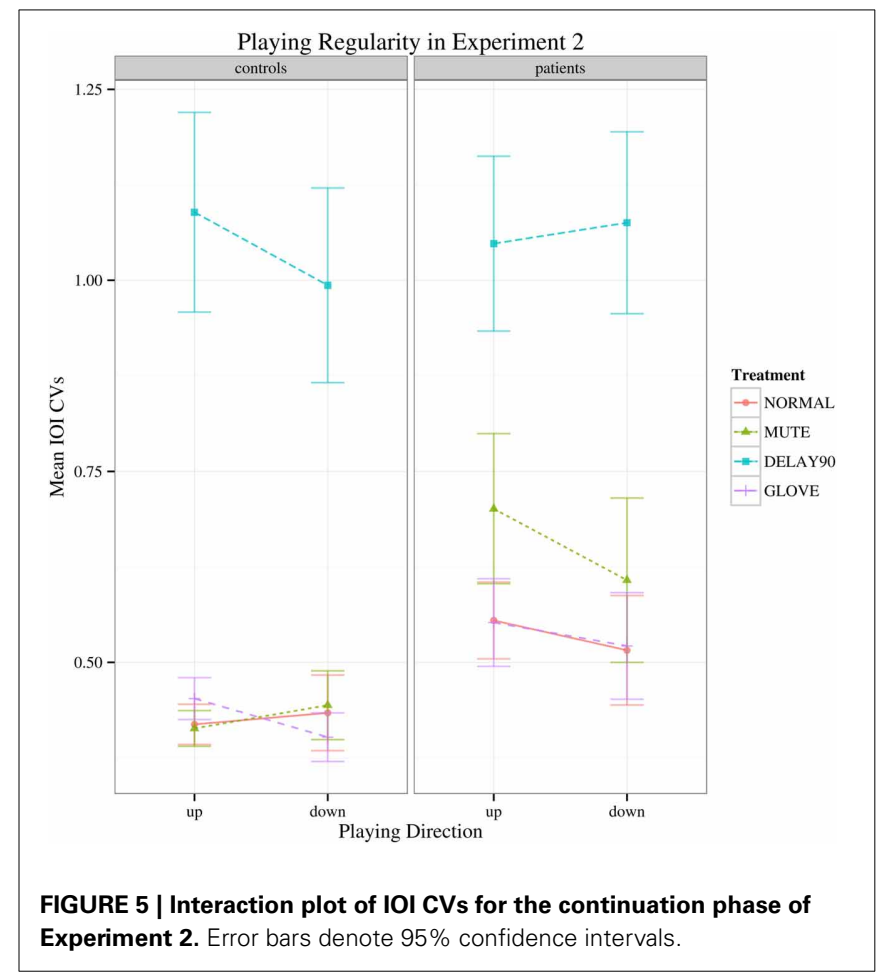

symptoms had slightly improved motor control under MUTE and only one patient had improved motor control under DELAY90 condition, while five patients had slightly improved motor control under GLOVE condition. Improved IOI CVs are marked in bold and italic fonts. Paired and one-sided $t$-tests of the IOI CVs calculated from each scale run were used to verify if the patient had significantly improved motor control under certain condition. For each patient, the NORMAL condition is tested against other altered sensory feedback conditions. The alternative hypothesis is that the true difference between the means is greater than 0 . Therefore, in Table 1, the altered sensory feedback conditions that show significantly reduced IOI CVs are the ones having $p<0.05$ and are underlined.

Compared to Experiment 1, both the healthy controls and the patients had markedly increased IOI CVs for the delay condition. This might be due to the effects of combined delayed auditory feedback plus pitch alteration are smaller than either kind of alteration on its own (Pfordresher, 2003). In Experiment 1, the duration of delay was $200 \mathrm{~ms}$, which was longer than the theoretical IOI of keypresses $(187.5 \mathrm{~ms})$, induced pitch shift relative to the keypress as well; whereas in Experiment 2, the duration of delay was $90 \mathrm{~ms}$, which was shorter than the theoretical IOI of keypresses, did not induce such pitch shift. Interestingly, in Experiment 2, there was no main effect of group while in Experiment 1, there was significant main effect of group. This stark contrast might be due to the different durations of the delay (200 ms for Experiment 1 and $90 \mathrm{~ms}$ for Experiment 2) and the different nature of the tasks (synchronization in Experiment 1 and continuation phase in Experiment 2). Playing with delayed auditory feedback in the continuation phase was much more difficult than in the synchronization phase, the healthy subjects showed 
Table 1 | Individual mean IOI CVs of all the patients in Experiment 2.

\begin{tabular}{lllll}
\hline Patient ID & Normal & Mute & Delay90 & Glove \\
\hline p01 & 0.63 & 0.635 & 1.245 & $\mathbf{0 . 5 9 5}$ \\
p02 & 0.29 & $\mathbf{0 . 2 7 5}$ & 1.005 & 0.335 \\
p03 & 0.315 & 0.485 & 0.855 & 0.32 \\
p04 & 0.435 & 0.465 & 0.955 & 0.49 \\
p05 & 0.445 & 0.61 & 1 & 0.45 \\
p06 & 0.315 & $\underline{\mathbf{0 . 2 8}}$ & 0.785 & $\underline{\mathbf{0 . 2 8 5}}$ \\
p07 & 0.57 & 0.63 & 0.92 & 0.61 \\
p08 & 0.495 & $\underline{\mathbf{0 . 3 6}}$ & 0.895 & $\underline{\mathbf{0 . 3 5 5}}$ \\
p09 & 0.62 & 0.825 & 1.445 & 0.57 \\
p10 & 0.335 & 0.34 & 0.84 & 0.315 \\
p11 & 0.275 & $\mathbf{0 . 2 5 5}$ & $\underline{\mathbf{0 . 2 1}}$ & 0.27 \\
p12 & 0.445 & 0.5 & 0.715 & $\underline{\mathbf{0 . 3 9}}$ \\
\hline
\end{tabular}

The conditions in which the patient showed less IOI CVs are marked with bold and italic fonts, and are underlined if $p<0.05$ according to the paired and one-sided t-tests.

significantly deteriorated fine motor control as well therefore there was no clear main effect of group.

\section{DISCUSSION}

In both Experiments 1 and 2, we found no difference between the behavior of healthy controls and patients. None of the AAF that we employed (either MUTE or DELAY of 200 and $90 \mathrm{~ms}$ ), and nor did the altered tactile feedback (GLOVE) improve the fine motor control of pianists suffering from MD. Taken together, the results are against our hypothesis, which assumed that the AAF or altered tactile feedback might serve as forms of successful sensory trick.

To the best of our knowledge, the only study that tested the role of auditory feedback in dystonia is a case study (Kojovic et al., 2012) in which a patient with generalized DYT1 dystonia showed dramatically improved symptoms while playing electric piano with auditory feedback. This improvement was reduced while the auditory feedback was masked but still noticeable. However, the reduction of dystonic symptoms in this case is considered different from typical sensory tricks, and is proposed to be more similar to paradoxical improvement (Fahn, 1989). What's more, primary generalized dystonia and focal dystonia differ in many respects, including the thresholds of sensory spatial discrimination (Molloy et al., 2003).

AAF has been extensively studied in speech and music performance related research. It has been shown that stuttering frequency can be significantly decreased by masking the auditory feedback (e.g., MacCulloch et al., 1970) or by delayed auditory feedback (e.g., Kalinowski et al., 1996), and delayed auditory feedback may serve as a treatment (Van Borsel et al., 2003). In music performance, auditory feedback has been shown to play a crucial role in the self-monitoring of music performance. It is shown that AAF can profoundly disrupt the performance of healthy professional musicians, and asynchronies between auditory feedback and actions primarily disrupt the timing of actions (Pfordresher, 2006). In the present study, AAF, especially delayed auditory feedback, disrupted the performance of both healthy pianists and pianists suffering from MD.
The MUTE condition can be seen as a task which directs the participants' attention toward their own movement. Theoretically speaking, the deprivation of auditory feedback would deautomatize the extensively trained auditory-motor coupling of expert musicians, thus recruit the structures outside the basal ganglia-supplementary motor area (SMA) system, such as the premotor cortex, which can only provide internally cued complex motor sequence during de-automatization (Alm, 2004). Nevertheless, only a few patients who had less severe symptoms participated in the current study showed mild benefit from such attentional shift, suggesting that the abnormal neural network involved in MD may not be normalized by this deautomatization, and it is reasonable to speculate that the more severe MD is more closely linked to deficient procedural memory, as is the case for several other movement disorders (Doyon, 2008). Although it has been shown that the functional cortical network in focal hand dystonia patients is impaired (Jin et al., 2011), the deprivation of auditory feedback in the long-trained coupling of sensorimotor cortical areas did not act as successful sensory trick for our patients. Instead, it has even been shown to have a detrimental effect on MD patient's fine motor control in the present study. The possible explanation for this finding is that playing scales under the MUTE condition relies heavily on an internal model that does not require auditory feedback, and the internal model has been shown to be impaired in MD (Ruiz et al., 2009, 2011; Lee et al., 2013).

The DELAY condition is essentially a task which manipulates the effect produced by participants' movements, which is similar to the studies that investigated the influence of the performer's focus of attention, which suggested that directing one's attention to the effects of the movements (external focus) involves different motor control processes than directing one's attention to one's own movements (internal focus) (McNevin et al., 2003). In case of movement disorders, it has been shown that patients with Parkinson's disease and a fall history can improve their balance with the adoption of an external focus (Landers et al., 2005). It should be noted that the sensory trick was once considered as a manoeuvre for distracting patients' attention (Abbruzzese and Berardelli, 2003). Nevertheless, in the current study, it is also shown that the manipulation of an external focus deteriorates the motor output and does not bring any benefit to the patients the way a successful sensory trick may do. It is interesting to note that the patients' playing was still affected by the manipulation of the external focus of attention similar to the healthy controls, implying the action and sound production are still strongly coupled in MD patients.

One of the studies that put an emphasis on the altered tactile feedback is a study carried out by Jabusch et al. (2011). Nevertheless, in this study, the authors addressed a different research question which aimed at the potential association between tactile sensory trick phenomenon ("glove effect" in this study) and the outcome after consequent treatment with botulinum toxin and/or pedagogical re-training. Furthermore, in this previous study, only $19 \%$ of patients showed significant improvement of fine motor control through wearing a glove in this study, and the patients who participated in this previous study had more severe symptoms (with median of standard 
deviations of inter-onset intervals of scale playing $=20.0 \mathrm{~ms}$ ) than our patients (median of standard deviations of inter-onset intervals of scale playing $=14.76 \mathrm{~ms}$ ). In the present study, we have smaller samples sizes ( 12 patients in both experiments) than the previous study. Both differences (degree of severity and sample size) could explain why in the current study there were no effects of glove on a group level. It should be mentioned, however, in the present study several individuals suffering from focal dystonia benefitted from glove condition. This points toward the heterogeneity in neural and/or behavioral organization of MD.

Behavioral studies on the sensory functions in dystonia is gaining its importance in light of providing effective strategies for recovery and understanding the underlying mechanisms (Tinazzi et al., 2009). In MD, although it has been shown that patients have deficits in temporal judgment, it was not clear how this sensory deficit can affect their playing ability under a disrupted sensory feedback network. Although the starting point of this study was the reports related to pipe organs having delayed auditory feedback, the present study provides evidence showing that the defected sensory network could not be normalized by the AAF conditions used in this experiment or altered tactile feedback such as wearing the glove. We hope that these results can contribute into further research in the sensory aspects of focal task-specific dystonia and help us to provide the patients with correct information and guide the patients with the most efficient therapeutic options.

\section{ACKNOWLEDGMENTS}

We wish to thank Mr. Aguirre, Mr. Hagemann, and Mr. Neubauer for programming the MIDI recording and manipulation program and the reviewers for the constructive comments.

\section{REFERENCES}

Abbruzzese, G., and Berardelli, A. (2003). Sensorimotor integration in movement disorders. Mov. Disord. 18, 231-240. doi: 10.1002/mds.10327

Alm, P. A. (2004). Stuttering and the basal ganglia circuits: a critical review of possible relations. J. Commun. Disord. 37, 325-369. doi: 10.1016/j.jcomdis.2004.03.001

Bangert, M., Peschel, T., Schlaug, G., Rotte, M., Drescher, D., Hinrichs, H., et al. (2006). Shared networks for auditory and motor processing in professional pianists: evidence from fMRI conjunction. Neuroimage 30, 917-926. doi: 10.1016/j.neuroimage.2005.10.044

Byl, N., Wilson, F., Merzenich, M., Melnick, M., Scott, P., Oakes, A., et al. (1996). Sensory dysfunction associated with repetitive strain injuries of tendinitis and focal hand dystonia: a comparative study. J. Orthop. Sports Phys. Ther. 23, 234-44. doi: 10.2519/jospt.1996.23.4.234

Chen, R., and Hallett, M. (1998). Focal dystonia and repetitive motion disorders. Clin. Orthop. Relat. Res. 351, 102-106. doi: 10.1097/00003086-199806000-00014

Downie, A. W., Low, J. M., and Lindsay, D. D. (1981). Speech disorder in parkinsonism-usefulness of delayed auditory feedback in selected cases. Int. J. Lang. Commun. Disord. 16, 135-139. doi: 10.3109/13682828109011394

Doyon, J. (2008). Motor sequence learning and movement disorders. Curr. Opin. Neurol. 21, 478-483. doi: 10.1097/WCO.0b013e328304b6a3

Fahn, S. (1989). Clinical variants of idiopathic torsion dystonia. J. Neurol. Neurosurg. Psychiatry 52(Suppl.), 96-100. doi: 10.1136/jnnp.52.Suppl.96

Finney, S. A., and Warren, W. H. (2002). Delayed auditory feedback and rhythmic tapping: evidence for a critical interval shift. Percept. Psychophys. 64, 896-908. doi: 10.3758/BF03196794

Gates, A., Bradshaw, J. L., and Nettleton, N. C. (1974). Effect of different delayed auditory feedback intervals on a music performance task. Percept. Psychophys. 15, 21-25. doi: 10.3758/BF03205822
Gentil, M. (1993). Speech rehabilitation in dysarthria. Folia Phoniatrica et Logopaedica 45, 31-35. doi: 10.1159/000266207

Holm, S. (1979). A simple sequentially rejective multiple test procedure. Scand. J. Stat. 6, 65-70.

Jabusch, H.-C., Paulig, J., Großbach, M., Boullet, L., and Altenmüller, E. (2011). Improved motor control after alteration of somatosensory input: prognostic value of "glove effect" in pianists with dystonia. Int. Symp. Perform. Sci. 11-16.

Jabusch, H.-C., Vauth, H., and Altenmüller, E. (2004). Quantification of focal dystonia in pianists using scale analysis. Mov. Disord. 19, 171-180. doi: $10.1002 / \mathrm{mds} .10671$

Jin, S.-H., Lin, P., and vHallett, M. (2011). Abnormal reorganization of functional cortical small-world networks in focal hand dystonia. PLOS ONE 6:e28682. doi: 10.1371/journal.pone.0028682

Kalinowski, J., Stuart, A., Sark, S., and Armson, J. (1996). Stuttering amelioration at various auditory feedback delays and speech rates. Int. J. Lang. Commun. Disord. 31, 259-269. doi: 10.3109/13682829609033157

Kojovic, M., Pareés, I., Sadnicka, A., Kassavetis, P., Rubio-Agusti, I., Saifee, T. A., et al. (2012). The brighter side of music in dystonia. Arch. Neurol. 69, 917-919. doi: 10.1001/archneurol.2012.33

Landers, M., Wulf, G., Wallmann, H., and Guadagnoli, M. (2005). An external focus of attention attenuates balance impairment in patients with Parkinson's disease who have a fall history. Physiotherapy 91, 152-158. doi: 10.1016/j.physio.2004.11.010

Lee, A., Furuya, S., Karst, M., and Altenmüller, E. (2013). Alteration in forward model prediction of sensory outcome of motor action in Focal Hand Dystonia. Front. Hum. Neurosci. 7:172. doi: 10.3389/fnhum.2013.00172

Lim, V. K., Bradshaw, J. L., Nicholls, M., and Altenmller, E. (2003). Perceptual differences in sequential stimuli across patients with musician's and writer's cramp. Mov. Disord. 18, 1286-1293. doi: 10.1002/mds.10528

MacCulloch, M. J., Eaton, R., and Long, E. (1970). The long term effect of auditory masking on young stutterers. Br. J. Disord. Commun. 5, 165-173. doi: $10.3109 / 13682827009011514$

McNevin, N. H., Shea, C. H., and Wulf, G. (2003). Increasing the distance of an external focus of attention enhances learning. Psychol. Res. 67, 22-29. doi: 10.1007/s00426-002-0093-6

Molloy, F. M., Carr, T. D., Zeuner, K. E., Dambrosia, J. M., and Hallett, M. (2003). Abnormalities of spatial discrimination in focal and generalized dystonia. Brain 126, 2175-2182. doi: 10.1093/brain/awg219

Naumann, M., Magyar-Lehmann, S., Reiners, K., Erbguth, F., and Leenders, K. L. (2000). Sensory tricks in cervical dystonia: perceptual dysbalance of parietal cortex modulates frontal motor programming. Ann. Neurol. 47, 322-328. doi: 10.1002/1531-8249(200003)47:3<322::AID-ANA7>3 3.CO; $2-5$

Oldfield, R. C. (1971). The assessment and analysis of handedness: the edinburgh inventory. Neuropsychologia 9, 97-113. doi: 10.1016/0028-3932(71)90067-4

Pfordresher, P. Q. (2003). Auditory feedback in music performance: evidence for a dissociation of sequencing and timing. J. Exp. Psychol. Hum. Percept. Perform. 29:949. doi: 10.1037/0096-1523.29.5.949

Pfordresher, P. Q. (2006). Coordination of perception and action in music performance. Adv. Cogn. Psychol. 2, 183-198. doi: 10.2478/v10053-008-0054-8

Pfordresher, P. Q., and Dalla Bella, S. (2011). Delayed auditory feedback and movement. J. Exp. Psychol. Hum. Percept. Perform. 37, 566. doi: 10.1037/a0021487

Pfordresher, P. Q., and Palmer, C. (2002). Effects of delayed auditory feedback on timing of music performance. Psychol. Res.66, 71-79. doi: $10.1007 / \mathrm{s} 004260100075$

Repp, B. H. (1999). Detecting deviations from metronomic timing in music: effects of perceptual structure on the mental timekeeper. Percept. Psychophys. 61, 529-548. doi: 10.3758/BF03211971

Robinson, A. P., and Froese, R. E. (2004). Model validation using equivalence tests. Ecol. Modell. 176, 349-358. doi: 10.1016/j.ecolmodel.2004.01.013

RStudio. (2013). RStudio: Integrated development environment for R (Version 0.97.551)

Ruiz, M. H., Jabusch, H. C., and Altenmüller, E. (2009). Detecting wrong notes in advance: neuronal correlates of error monitoring in pianists. Cereb. Cortex 19, 2625-2639. doi: 10.1093/cercor/bhp021

Ruiz, M. H., Strübing, F., Jabusch, H. C., and Altenmüller, E. (2011). EEG oscillatory patterns are associated with error prediction during music performance and are altered in musician's dystonia. Neuroimage 55, 1791-1803. doi: 10.1016/j.neuroimage.2010.12.050 
Schramm, A., Reiners, K., and Naumann, M. (2004). Complex mechanisms of sensory tricks in cervical dystonia. Mov. Disord. 19, 452-457. doi: $10.1002 / \mathrm{mds} .10689$

Serrien, D. (2008). The neural dynamics of timed motor tasks: evidence from a synchronization-continuation paradigm. Eur. J. Neurosci. 27, 1553-1560. doi: 10.1111/j.1460-9568.2008.06110.x

Sokal, R. R., and Rohlf, F. J. (1995). Biometry, 3rd Edn. New York, NY: WH Freman and company

Stevens, L. T. (1886). On the time-sense. Mind 43, 393-404. doi: 10.1093/mind/osXI.43.393

Suttrup, I., Oberdiek, D., Suttrup, J., Osada, N., Evers, S., and Marziniak, M. (2011). Loss of sensory function in patients with idiopathic hand dystonia. Mov. Disord. 26, 107-113. doi: 10.1002/mds.23425

Team, R. D. C. (2005). R: A Language And Environment For Statistical Computing. Vienna: R Foundation for Statistical Computing, ISBN 3-900051-07-0. Available online at: http://www. R-project.org.

Tinazzi, M., Fiorio, M., Fiaschi, A., Rothwell, J. and Bhatia, K. (2009). Sensory functions in dystonia: insights from behavioral studies. Mov. Disord. 24, 1427-1436. doi: $10.1002 / \mathrm{mds} .22490$

Van Borsel, J., Reunes, G., and Van den Bergh, N. (2003). Delayed auditory feedback in the treatment of stuttering: clients as consumers. Int. J. Lang. Commun. Disord. 38, 119-129. doi: 10.1080/1368282021000042902
Zatorre, R. J., Chen, J. L., and Penhune, V. B. (2007). When the brain plays music: auditory-motor interactions in music perception and production. Nat. Rev. Neurosci. 8, 547 558. doi: 10.1038/nrn2152

Conflict of Interest Statement: The authors declare that the research was conducted in the absence of any commercial or financial relationships that could be construed as a potential conflict of interest.

Received: 15 March 2013; paper pending published: 07 April 2013; accepted: 28 November 2013; published online: 17 December 2013.

Citation: Cheng FP-H, Großbach M and Altenmüller EO (2013) Altered sensory feedbacks in pianist's dystonia: the altered auditory feedback paradigm and the glove effect. Front. Hum. Neurosci. 7:868. doi: 10.3389/fnhum.2013.00868 This article was submitted to the journal Frontiers in Human Neuroscience.

Copyright (c) 2013 Cheng, Großbach and Altenmüller. This is an open-access article distributed under the terms of the Creative Commons Attribution License (CC BY). The use, distribution or reproduction in other forums is permitted, provided the original author(s) or licensor are credited and that the original publication in this journal is cited, in accordance with accepted academic practice. No use, distribution or reproduction is permitted which does not comply with these terms. 